\title{
Nanotechnology-Based Therapies for Skin Wound Regeneration
}

\author{
Ilaria Tocco, ${ }^{1}$ Barbara Zavan, ${ }^{2}$ Franco Bassetto, ${ }^{1}$ and Vincenzo Vindigni ${ }^{1}$ \\ ${ }^{1}$ Institute of Plastic Surgery, University Hospital of Padova, 35128 Padova, Italy \\ ${ }^{2}$ Department of Histology, Microbiology and Biomedical Technologies, University of Padova, 35128 Padova, Italy
}

Correspondence should be addressed to Ilaria Tocco, toccoilaria@gmail.com

Received 8 February 2012; Revised 19 February 2012; Accepted 24 February 2012

Academic Editor: Xiaoming Li

Copyright (C) 2012 Ilaria Tocco et al. This is an open access article distributed under the Creative Commons Attribution License, which permits unrestricted use, distribution, and reproduction in any medium, provided the original work is properly cited.

\begin{abstract}
The cutting-edge combination of nanotechnology with medicine offers the unprecedented opportunity to create materials and devices at a nanoscale level, holding the potential to revolutionize currently available macroscale therapeutics. Nanotechnology already provides a plethora of advantages to medical care, and the success of nanoparticulate systems suggests that a progressive increase in the exploration of their potential will take place in the near future. An overview on the current applications of nanotechnology to wound healing and wound care is presented.
\end{abstract}

\section{Introduction}

Nobel Prize winner Richard Feynman was the first in 1959 to predict the future emergence of a new science which could be able to deal with structures on a scale $1-100 \mathrm{~nm}$. Fifty years after, the unpredictable result is the ability to manipulate materials on the same unimaginably small scale which is used by nature [1]. The repercussions that nanotechnology is having in our lives nowadays are towering: the current applications to molecular biology are leading to the development of structures, devices, and systems bearing a capacity to revolutionize medical therapeutics and diagnostics, which has never been seen before.

Nanodevices are innovative and can provide a wide range of advantages: from the ability of nanoparticles to enter into the cytoplasmic space across cellular barriers, like Trojan horses, and activate specific transport mechanisms [2,3]; to the modulation of drugs biocompatibility, bioavailability and safety profiles through nanodelivery systems [4]. Furthermore, therapeutic selection can increasingly be tailored to each patient's profile. Nanotherapies represent a great opportunity to enhance currently available medical treatments, improving standard care and prognosis for challenging healthcare issues, like impaired wound healing.

The public health impact of chronic wounds is staggering. An estimated 1.3 to 3 million US individuals are believed to have pressure ulcers and as many as $10 \%$ to $15 \%$ of the 20 million individuals with diabetes are at risk of developing diabetic ulcers [5]. Nonhealing wounds are the results of a stop in the progression of the normal sequence of cellular and biochemical events towards the restoration of the skin's integrity. Factors delaying wound healing include either preexisting comorbidities (diabetes, chronic peripheral vasculopathy, and immunosuppression), leading to lack of appropriate metabolism and clearance from toxic substances of the wound, and/or sudden complications, such as infections, exalting the inflammatory state. Nanobiotechnology combined with the knowledge on cellular and subcellular dysfunctional events occurring in delayed wound healing offers great opportunities for improving wound care.

This paper will focus on the currently available nanotechnology-based therapies in the field of wound healing and skin regeneration. To appreciate the importance of the functions of the innovative nanosystems, an understanding of the wound healing process is essential.

1.1. The Wound Healing Process. The wound healing process is classically defined as a series of continuous, sometimes overlapping, events. These are haemostasis, inflammation, proliferation, epithelisation, maturation, and remodelling of the scar tissue [6] (Figure 1).

Haemostatic events occur immediately after injury. The exposure of subendothelial collagen and the formation of thrombin lead to the activation of the platelets, located in 


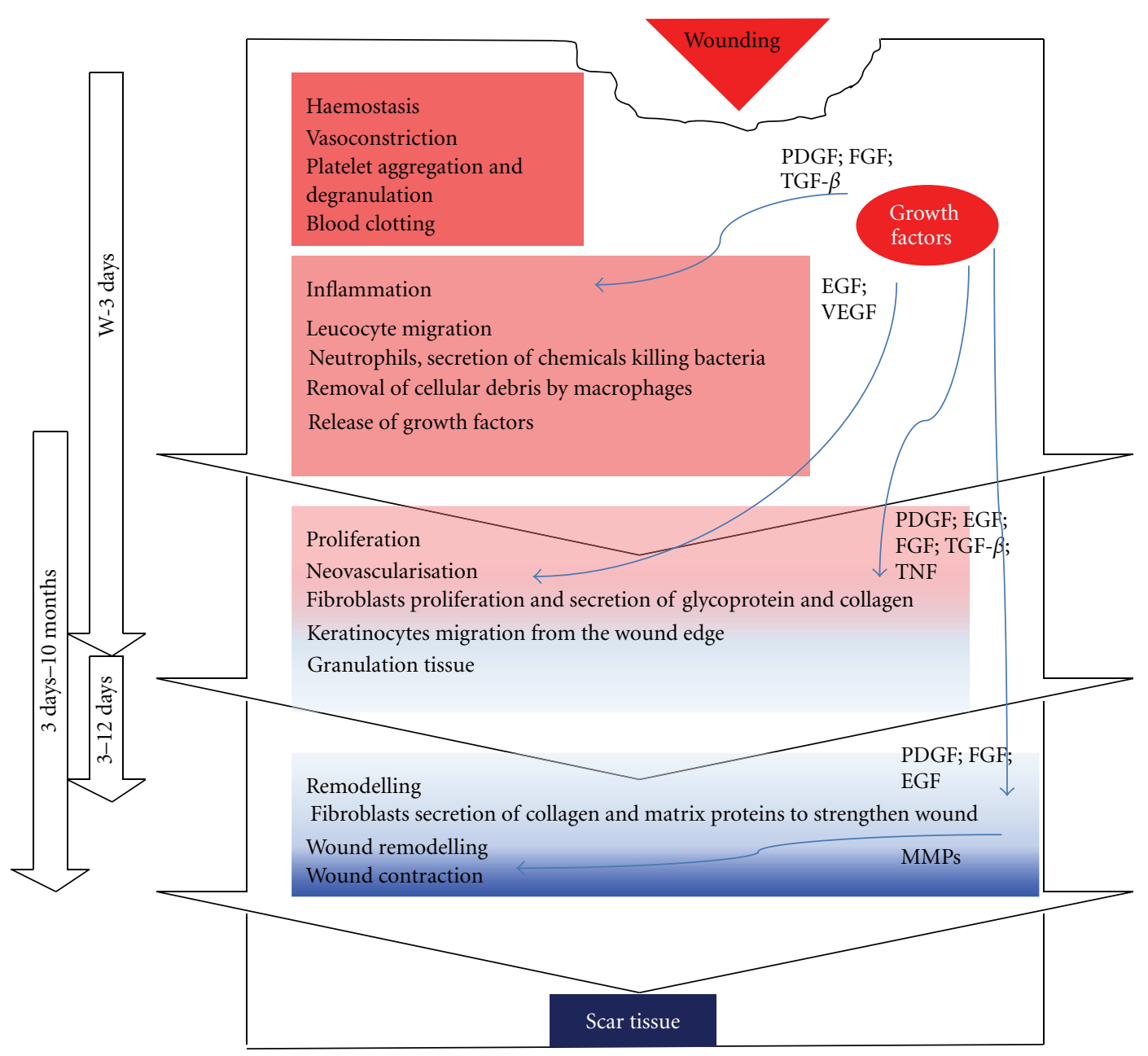

Figure 1: The main biological phases in wound healing. The events in wound healing and the soluble factors involved in each phase are well defined. Although presented as separated for absolute clarity, no phase is initiated exactly at the complexion of the previous one, and all phases overlap to a certain degree (see arrows on the left).

the intravascular space. Activated platelets play a trigger role in a number of events:

(i) activation of the coagulation cascade. This eventually leads to the formation of a fibrin clot that acts as scaffolding for other cells that later enter the wound;

(ii) activation of the complement system;

(iii) platelet degranulation: cells release an array of cytokines, growth factors, and vasoactive substances from the platelet $\alpha$-granules, such as platelet-derived growth factor (PDGF), transforming growth factor $\beta$ (TGF- $\beta$ ), fibroblast growth factor (FGF), endothelial growth factor (EGF), platelet-derived angiogenesis factor, serotonin, bradykinin, platelet-activating factor, thromboxane A2, platelet factor IV, prostaglandins, and histamine. These platelet releasates initiate the early events of wound healing $[6,7]$.

The inflammatory phase begins immediately after injury and may continue for up to 6 days [8]. Growth factors released from the platelets diffuse into tissues surrounding the wound and chemotactically draw inflammatory cells into the injured area. Neutrophils are the first inflammatory cells to enter the wound, followed by monocytes. Once chemotaxis is completed, local mediators activate the inflammatory cells. Activated neutrophils release a number of lysosomal enzymes (such as elastase, neutral proteases, and collagenase) which proteolytically remove damaged components of extracellular matrix (ECM) [8]. Activated monocytes acquire the macrophage phenotype and aid in host defence [8].

The proliferation phase is characterised by the formation of the ECM and the beginning of angiogenesis. The primary cells involved in this phase are fibroblasts and endothelial cells. They proliferate in response to growth factors and cytokines that are released from macrophages, platelets and mesenchymal cells, or have been stored in the fibrin clot. In addition to chemotactically drawing fibroblasts into the wound, PDGF, FGF, and EGF induce fibroblast activation and proliferation [9]. During the first 2-3 days after-injury, fibroblasts activity predominantly involves migration and proliferation. After this time, fibroblasts release collagen and glycosaminoglycans (mainly hyaluronic acid, chondroitin4-sulphate, dermatan sulphate, and heparin sulphate) in 
response to macrophage-released growth factors, hypoxia and by-products of anaerobic metabolism. The combination of collagen and fibronectin forms the new ECM, which is essential for the development of granulation tissue that eventually fills the wound [6]. Angiogenesis accompanies fibroblast proliferation and allows nutrients and healing factors to enter the wound space. It is also essential for the growth of granulation tissue. The principle growth factors that regulate angiogenesis are FGF, released by damaged endothelial cells and macrophages, and vascular endothelial growth factor (VEGF) which is released by keratinocytes and macrophages [6].

The maturation phase usually begins 3 weeks after injury and can take up to 2 years to complete [10]. Unlike uninjured skin, the arrangement of newly formed collagen fibres in the wound is random and disorganised. The remodelling of collagen fibres into a more organised lattice structure gradually increases the tensile strength of the scar tissue, though this never exceeds 80 percent of the strength of intact skin. Remodelling of the ECM involves a balance between collagen synthesis and degradation, which is operated by several enzymes, like matrix metalloproteinases (MMPs), neutrophilreleased elastase and gelatinase, collagenases and stromelysins [11].

Wounds that do not heal within three months are considered chronic. In acute wounds, there is a precise balance between production and degradation of molecules such as collagen; in chronic wounds this balance is lost and degradation plays too large a role. Chronic wound bed has been demonstrated to differ from acute wounds for a higher concentrations of proteases (such as MMPs) [12] and lower levels of growth factors and cytokines [13]. A high and prolonged proteolytic activity may lead to the degradation of growth factors, detaining the wound in the inflammatory stage for too long [14].

\section{Nanotherapies for Wound Healing}

An increasing number of products emerging from the application of nanotechnology to the science of wound healing is currently under clinical investigation. The current nanoscale strategies, both carrier, drug related and scaffold (Figure 2), that target the main phases of wound repair will be discussed, highlighting the cellular signals involved (Table 1).

2.1. Nanoparticle-Bearing Endogenous Molecules. As we pointed out previously, soluble active proteins (cytokines and growth factors) govern the progression of the healing phases through the modulation of the cellular and molecular components involved [43]. Because of the control they exert on wound repair, bioactive proteins have gained progressive interest for the treatment of chronic dehiscent wounds. Clinical trials involving the application of exogenous recombinant growth factors to chronic wounds have been conducted for the past 10 years in anattempt to find a way to accelerate healing [44]. Although the results of several pilot trials have been encouraging, the overall results have been somewhat discouraging. To date, only a single recombinant growth factor-recombinant human PDGF-BB-has been approved by the United States Food and Drug Administration, and only for use in diabetic foot ulcers [45]. In fact, the major limitation to the topical use of growth substances is that in human plasma the half-life of proteins involved in signalling cascades is very short (few seconds) due to close control and inactivation by protease inhibitors [46]. Therefore, the clinical use of endogenous factors as pure compounds is limited by the breakdown by the proteolytic enzymes that enrich the wound site [47]. Several attempts have been already made to provide biological molecules long-term protection from enzymatic degradation (liquids, gels, collagen sponges, and gene transfer). Recently, the development of nanoscale systems for drug delivery has opened new possibilities to enhance the biological efficacy of molecules through a controlled release for extended periods of time [48]. Endogenous active molecules that have been engineered nowadays include thrombin, nitric oxide, growth factors, opioids, and protease inhibitors.

Thrombin (also termed activated factor II or factor IIa) is a protein involved in the final stage of the coagulation cascade activated by wounding. In addition to its well-documented role in the formation of fibrin clots and platelet activation, thrombin has direct effects on inflammatory cells, fibroblasts, and endothelial cells: it stimulates chemotaxis and aggregation of neutrophils, lymphocytes, and monocytes cells, and the proliferation of fibroblasts, epithelial and endothelial cells $[49,50]$. Thus, thrombin may play an important role in initiating early cellular events in tissue repair [49]. Since 1988 researchers suggested that thrombin receptor-activating peptides could be useful in vivo to mimic the natural effects of thrombin interaction with receptors on various types of cells [51], a delivery systems that could provide thrombin a long-term protection from its natural inhibitors (antithrombin and activated protein C) has been pursued. In recent years, nanobiotechnology has provided the means to enhance the bioavailability of thrombin by conjugation with iron oxide nanoparticles ( $\gamma-\mathrm{Fe}_{2}-\mathrm{O}_{3}$ conjugation) [15]. Animal models were tested for wound response to the treatment with conjugated thrombin: on a 28 -day treated wound, results obtained analysing tensile strength indicated a significant acceleration of healing process when compared with free-thrombin-treated wounds and untreated wounds. Obtaining a greater tensile strength may potentially reduce surgical complications such as wound dehiscence.

Nitric oxide (NO) is a small radical, formed from the amino acid L-arginine by three distinct isoforms of nitric oxide synthase. The inducible isoform (iNOS) is synthesized in the early phase of wound healing by inflammatory cells, mainly macrophages. However, many cells participate in NO synthesis during the proliferative phase after wounding. NO regulates collagen formation, cell proliferation, and wound contraction in distinct ways in animal models of wound healing [52]. NO is also a well-known antimicrobial agent, interfering directly with DNA replication and cell respiration through the inactivation of zinc metalloproteins [53]. Treatment of acute and chronic wound failure with $\mathrm{NO}$ has been for years a major unresolved goal. Attempts at novel nitric 

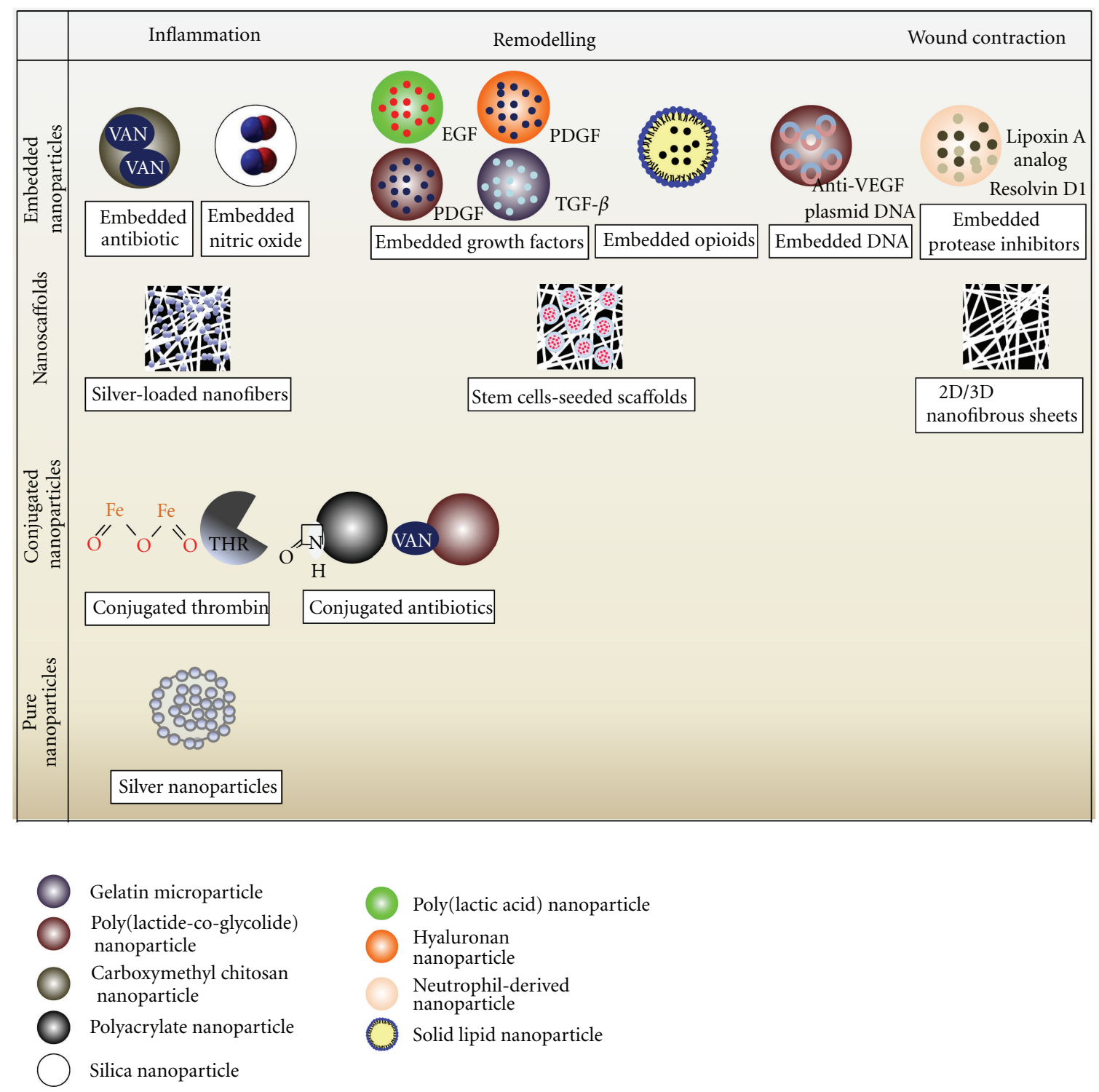

Poly(lactic acid) nanoparticle

Hyaluronan

nanoparticle

Neutrophil-derived

nanoparticle

Solid lipid nanoparticle

FIGURE 2: Nanostrategies currently in use for promoting skin wound healing. In the main panel, nanostrategies currently in use to improve healing are illustrated. The therapeutic potential of nanosphere-based strategies and nanoscaffolds is emphasized through correlation with the healing phase to which the biological action is targeted. In the right panel, materials explored for nanoparticulate-delivery systems are listed. Two- and three-dimensional nanofibrous sheets can be made of both degradable (collagen and chitosan) and nondegradable (PLA, PLACL, polyurethane, and PVA polymers) nanofibers.

oxide therapies, in the form of nitric oxide donors, have shown limited potential in treating cutaneous infection [54]. A novel approach is offered by recently engineered nanoporous materials, that make possible the storage and delivery of small gaseous short-lived NO, allowing the free radicals to exert their antibacterial activity. Using silane hydrogel-based nanotechnology [23], NO remains trapped and stable within a dry matrix until the matrix is exposed to moisture. The dry matrix allows for NO nanoparticles to be easily stored and applied. Once exposed to moisture, the drug is released from the nanoparticle over an extended period of time at a relatively fixed concentration. This sustained release distinguishes nanoparticles from other vehicles, such as injections, that release a large concentration of the drug with a rapid return to baseline. The ease of storage, application, and the ability to alter release rate and concentration with minimal risk of toxicity make this powder formulation ideal for cutaneous delivery. Authors showed promising results from NO-releasing nanodelivery systems in preventing and treating skin infections caused by methicillinresistant Staphylococcus aureus (MRSA), which is one of the major causes of hospital-acquired infections [55, 56]. Moreover, NO-releasing silica nanomolecules have been demonstrated to exert a bactericidal activity against Pseudomonas aeruginosa [57] and Acinetobacter baumannii [58]: both microbial agents have become an increasingly prevalent cause of hospital-acquired infections during the last 15 years, the majority of clinical A. baumannii 
TABLE 1: Executive summary table.

Inflammation

(i) thrombin is one of the first products of the coagulation cascade occurring during haemostasis, and is responsible for platelet activation and aggregation, leading to the formation of the "platelet plug" and allowing cells and fluid to enter the wound bed. In human plasma, thrombin is rapidly degraded $(15 \mathrm{sec})$. In order to provide long-term protection, it has been conjugated with iron-oxide nanoparticles for treatment of incisional wounds in rats [15]

(ii) bacterial infection and sepsis exacerbate the inflammatory state and cause tissue damage

(a) nanoparticles bearing vancomycin or $\mathrm{N}$-methyltiolated $\beta$ lactams have been developed to act against wound contamination by MRSA [16-19]

(b) silver-based nanoparticles were developed to take advantage on the multilevel antibacterial action of silver and try to reduce the development of microbial resistance. Pure biostable nanoparticles were produced through photoassisted reduction and ion stabilization. Silver nanoparticles were also loaded into nanofibers [20]. A direct promotion of wound healing by silver nanoparticles through reduction of the cytokine-modulated inflammation and cell migration and proliferation was also demonstrated [21, 22]

(c) donor NO silica nanoparticles showed speed healing by killing both Gram-positive and Gram-negative bacteria and overcoming the NO deficiency [23]. No-releasing nanoparticles may also potentially accelerate healing by a promotion of angiogenesis and tissue remodelling [24]

\section{Proliferation}

(i) the aim of growth factors is to promote cell migration into the wound site, stimulate the growth of epithelial cells and fibroblasts, start the formation of new blood vessels, and profoundly influence the remodelling of the scar. To enhance the in vivo efficacy of growth factors they have been incorporated into polymer nanocarriers to sustain release. PLA/PLGA/PEG/hyaluronan/gelatin nanoparticles embedded with different growth factors have been successfully applied on skin wounds [25-31]

(ii) opioids have been recently indicated as factors promoting keratinocytes migration. Solid lipid nanoparticles were embedded with opioids, confirming the influence of these drugs on keratinocytes migration $[32,33]$

(iii) nanofibrous scaffolds: electrospunnanofibers networks support cell adhesion, proliferation, and differentiation mimicking the fibrous architecture of the extracellular matrix. Both degradable (collagen and chitosan) and nondegradable (PLA, PVA, PLACL, and polyurethane) fibers are used for 2D and 3D constructs [34-36]. Scaffolds were also engineered to contain growth factors-releasing nanoparticles enhancing wound repair [37]

\section{Remodelling}

(i) matrix metalloproteinase collagenolytic activity appears to be upregulated in chronic wounds. Protease inhibitors were loaded into human derived nanoparticles, showing a proresolving action and accelerated healing [38]

(ii) gene therapy: nonviral polymeric gene delivery systems offer increased protection from nuclease degradation, enhanced plasmid DNA uptake, and controlled dosing to sustain the duration of plasmid DNA administration. Gene delivery systems are formulated from PLGA polymers, polysaccharides, and chitosan [39-41]

(iii) stem cells: cell-based therapies hold the potential to promote vascularization and tissue regeneration. The hVEGF gene was delivered through biodegradable polymeric nanoparticles: treated stem cells showed the engraftment of the tissue [42]

isolates displaying high-level resistance to common antibiotics [59]. Also, the beneficial effects of $\mathrm{NO}$ seem to overcome the antimicrobial activity and involve directly the healing process. Experiments demonstrated that pharmacological or genetic (NOS knockout) reduction of NO impairs the speed and effectiveness of wound healing, and that this process can be reversed by restoring NO production with increased NOS substrate (arginine), or by transfecting with the missing NOS gene [24]. Thus, application of NOreleasing nanoparticles may potentially accelerate healing not only by killing bacteria but also by a promotion of angiogenesis and tissue remodelling.

The clinical use of growth factors in wound healing has been of great interest recently. They have the potential to accelerate the healing process by attracting cells into the wound site (TGF- $\beta$ ), promoting cell migration, stimulating the proliferation of epithelial cells and fibroblasts (FGF and PDGF), as well as initiating the formation of new blood vessels (FGF and VEGF), and finally participating in the remodelling of the scar [46]. A mean of enhancing the in vivo efficacy of growth factors, preventing degradation from proteolytic enzymes, is to stabilize protein structure and biological activity, prolonging the length of time over which growth factors are released at the delivery site $[60,61]$. The period of drug release from a polymer matrix can be regulated by drug loading, type of polymer used and the processing conditions. Delivery systems have been designed in a variety of configurations and have been fabricated from different types of natural and synthetic polymers, both degradable and non degradable. Poly(lactic acid) (PLA) and poly(lactic-co-glycolic acid) (PLGA) have been demonstrated to be biocompatible and biodegradable suitable materials. To achieve long-term in vivo circulation, the surface is generally modified with polyethylene glycol (PEG), reducing the clearance by the reticuloendothelial system [25]. In biodegradable carriers, growth factor release is controlled by the polymer matrix's rate of degradation, which causes changes in the morphological characteristics of the materials, such as porosity and permeability [26]. Particulate delivery systems explored involve TGF- $\beta$-embedded gelatine microparticles [27], EGF in PLA microspheres [28], FGF in gelatin microspheres [29], and PDGF-embedded PLGA nanospheres [30]. In general, 
porous materials seem to retain a higher specific surface for the adsorption and release of active components and enhancement of drug release. Nevertheless, the particles show some limits which are associated with the use of organic solvents in the production process, leading to pollution and toxicity of the product [62]. Therefore, alternative techniques of fabrications have been proposed, such as spray drying, which is based on the use of supercritical fluids (especially $\mathrm{CO}_{2}$ ) and offers the advantages of being environmentally safe and inexpensive [63]. A notable recent application of this technology for the delivery of growth factors in vivo was offered by Zavan et al. [31]: hyaluronan-based (HYAFF11) porous nanoparticles were embedded with PDGF as a system designed for the in vivo treatment of skin ulcers. PDGF is known since 1986 to successfully promote chronic wounds healing through a stimulation of chemotaxis, proliferation and ECM deposition [64]. HYAFF11, the benzyl ester of hyaluronic acid, is a biopolymer that has found numerous applications for in vitro reconstruction of skin as well as for the in vivo regeneration of small arteries and veins [65]. HYAFF particles have the ability to absorb growth factors and to release them in a temporally and spatially specific event-driven manner. This timed and localized release of PDGF promoted optimal tissue repair and regeneration of full-thickness wounds.

Beside the dedicated growth factors, opioids have been recently indicated as factors promoting keratinocytes migration [66]. This finding is of great interest because of a possible enhancement of wound healing through topical applications of opioids at the wound site for pain reduction. Conventional formulations failed to consistently provide sufficient pain control in patients, possibly due to local drug degradation or insufficient concentrations at the target site [32]. Since long intervals for painful wound dressing changes are intended, the formulations should allow for prolonged opioid release. Bigliardi et al. [32] first developed nanoparticulate carriers to increase opioids skin penetration and slow the release of the loaded drug. Experiments on human keratinocyte-derived cell line HaCaT showed that opioids stimulated cell migration and closure of experimental wounds. Enhancement of migration was concentration-dependent and could be blocked by the opioid receptor antagonist naloxone, indicating a specific opioid-receptor interaction. Küchler followed [33] demonstrating that morphine-loaded solid lipid nanoparticles accelerated reepithelialisation on a human-based $3 \mathrm{D}$ wound healing model. On standardised wounds, keratinocytes almost completely covered the dermis equivalent after 4 days, which was not the case when applying the unloaded particles. In conclusion, acceleration of wound closure, low cytotoxicity, and irritation as well as possible prolonged morphine release make solid lipid nanoparticles an interesting approach for innovative wound management.

Matrix metallo proteinases (MMPs) are zinc-dependent endopeptidases that cleave most macromolecules within the ECM during the maturation of the wound [67]. The process of remodelling constitutes a balance between collagen production, breakdown, and remodelling. The biological activity of MMPs is strictly balanced by the presence of specific tissue inhibitors (TIMPs). A tightly coordinated expression of specific combinations of MMPs and TIMPs appears to be necessary for proper wound maturation. As previously stated, an uncontrolled proteolytic activity leads to delayed healing through degradation of growth factors. In light of these considerations, research approaches to improve the remodelling phase are directed to manage enzymatic activity of MMPs through the topical application of protease inhibitors. Norling et al. [38] recently took advantage of aspirin-triggered resolving D1 and lipoxin A4 analogs and developed a carrier providing stable biological activity to these natural compounds. Human-derived nanoparticles were enriched with the protease inhibitors, and wound healing reactions were tested in a murine model. Polymorphonuclear cell influx showed a dramatical reduction in treated wounds, with shortened resolution intervals, and a proresolving action that in the end accelerated healing.

2.2. Nanoparticle-Bearing Antibiotics. During the healing process, infection is an issue potentially compromising the final wound closure, exacerbating the tissue damage [68]. Nowadays, wound infection is no longer the ominous event as at the beginning of the 20th Century, when infection of wounds, especially burns, was the major cause of morbidity and mortality (over 50\%) [69]. Nevertheless, an appropriate antimicrobial therapy of the wound controlling colonization and proliferation of microbial pathogens, including multidrug-resistant organisms, is still required for an appropriate wound care. Staphylococcus aureusis one of the most common pathogens involved in wound infections. The pharmacological treatment encounters today severe limitations due to the development of antibiotic resistant strains. For example, penicillinase (an enzyme that breaks down the $\beta$-lactam ring of the penicillin molecule) is responsible for the resistance to penicillin of Staphylococcus aureus: the failed treatment of staphylococcal local infections leads to the onset of serious late complications (bacteraemia, sepsis, toxic shock syndrome) [69].

The delivery of antibiotic therapy via nanoparticles offers great potential advantages. Particularly, a controlled release would decrease the number of doses required to achieve the desired clinical effect, potentially reducing the risk of development of antibiotic resistance. The physiochemical properties of nanoparticulate drug delivery systems (size, surface charge, and nature) are determinant in vivo for the factors delivery. In particular, it is known that 20-200 nm particulates are suitable for delivery of therapeutics; larger size particles suffer from quick uptake by the reticulo-endothelial system and rapid clearance from circulation, whereas the smaller size will tend to cross the fenestration in the hepatic sinusoidal endothelium, leading to hepatic accumulation [70].

In the treatment of staphylococcal infections, the latest generation antibiotic and assumed to be the most useful at the moment is vancomycin. Several vancomycin-modified nanoparticles have been developed to enhance the pharmacokinetics and pharmacodynamics of the antibiotic molecule. Hachicha et al. [16] recently proposed the use of vancomycin-conjugated nanoparticles for intraocular continuous release injection for endophthalmitis prophylaxis. The drug concentration was proved to be maintained above 
the minimal inhibitory limit for 24 hours. Chakraborty et al. [17] developed carboxymethyl chitosan-based nanoparticles, which were loaded with vancomycin and proved effective against drug-resistant staphylococcal strains.

The effectiveness of nanobiotechnology in enhancing the therapeutic properties of antibiotic molecules is emphasized by the case of $\mathrm{N}$-methylthiolated $\beta$-lactams: these compounds have been recently identified and proved effective against Staphylococcus bacteria, including MRSA. These lactams exert growth inhibitory effects on bacteria through a mode of action that is distinctively different from other $\beta$ lactam antibiotics, with peculiar structure-activity patterns. Nevertheless, their potential application is limited by the exceeding low water solubility [18]. Thus, a methodology was developed by Turos et al. [19] to obtain an emulsion of polyacrylate nanoparticles in which the drug monomer was incorporated into the polymeric matrix: in vitro screens confirmed the nanoparticles to be nontoxic to human dermal fibroblasts and stable in blood serum for 24 hours.

Within the bacterial species, resistance to all known antibiotic classes has occurred due to mutation and horizontal gene transfer. This has led to anxiety regarding the futurea vailability of effective chemotherapeutic options. Due to the outbreak of infectious diseases caused by different pathogenic bacteria and the development of antibiotic resistance, the pharmaceutical companies and the researchers are now searching for new antibacterial agents.

2.3. Silver-Based Nanoparticles. The use of silver for the treatment of ulcers is reported since the 5th Century B.C. In the 17th and 18th centuries, silver nitrate was already used for ulcer treatment. The antimicrobial activity of silver was established in the 19th century. Nevertheless, after the introduction of antibiotics in 1940 the use of silver salts decreased. Subsequently, silver salts and silver compounds have been used in different biomedical fields, especially in burn treatment [71].

The antimicrobial activity of silver appears high: due to its multilevel (including multidrug resistance) antibacterial effects [72] and low systemic toxicity [73], it provides an antibacterial effect that considerably reduce the chances of developing resistance. Evidently, the greatest rate of silver ions release is wished in order to enhance the clinical effect and avoid the insurgence of silver-resistant mutant bacteria. Nanotechnology has provided the means of producing pure biostable silver nanoparticles, either through photoassisted reduction and ion stabilization or loading of the metallic particles into nanofibers [20]. In all cases, 7-20 nm silver nanoparticles exhibited antibacterial (especially anti-Gram negative) and antifungal activity, and they are also synergistic to common antibiotic therapy (streptomycin, kanamycin, and polymyxyn B) [20]. Studies in vivo demonstrated also a direct promotion of wound healing by silver nanoparticles through reduction of the cytokine-modulated inflammation: silverinduced neutrophils apoptosis, decreased MMPs activity, and negative modulation on TGF- $\beta$ resulted in an overall acceleration of wound healing rate and reduction on hypertrophic scarring [21]. Also, wound healing rate was demonstrated to be increased by silver ions by a promotion of proliferation and migration of keratinocytes along with a differentiation of fibroblasts into myofibroblasts, thereby promoting wound contraction [22].

Though the progressive expansion of the therapeutic application of silver nanoparticles, the use of a metallic compound carries possible side effects that must be taken into consideration. Studies have been investigating the biosafety of silver as a therapeutic agent, reporting an acceptable biocompatibility [74], though the occasional development of argyria (a cosmetic blue-grey coloration of the skin).

2.4. Nanoparticles and Gene Therapy. Polymeric gene delivery systems offer several advantages for plasmid DNA delivery, such as protection from degradation by the nuclease and controlled prolonged release. The potential retained by the modulation of gene expression in the process of wound healing lead researchers to apply for an engineered system for DNA transfection. Actually, transfection capability has been tested in vitro: biocompatible and biodegradable PLGA polymers [39] were engineered to obtain high plasmid loading efficiency [75] and then loaded withan antiangiogenic plasmid DNA (pFlt23k). The PLGA nanoparticles were prepared by a supercritical fluid extraction of emulsions based on $\mathrm{CO}_{2}$ : this allowed high loading of pDNA $(19.7 \%, \mathrm{w} / \mathrm{w})$, high loading efficiency ( $>98 \%$ ), and low residual solvents $(<50 \mathrm{ppm})$. The VEGF secretion by epithelial cells was significantly reduced, showing a potential value in treating wound disorders in which VEGF is elevated.

In the course of studies on nonviral DNA carriers for gene delivery and therapy other materials have been applied, like polysaccharides and other cationic polymers. Chitosan also was found to be particularly suitable, since it can promote long-term release of incorporated drugs [76]. Masotti and Ortaggi recently described a nanofabrication method that may be useful for obtaining small DNA-containing chitosan nanospheres $(38 \pm 4 \mathrm{~nm})$ for biomedical applications [40]. Their reported osmosis-based method has general applicability to various synthetic or natural biopolymers, resulting in nanostructured systems of different size and shape that may be used in several biotechnological applications. Chellat et al. also recently took advantage of the biochemical properties of chitosan to test DNA-loaded nanoparticles on a human macrophage cell line to study the potential modulation of the expression of proinflammatory cytokines, metalloproteinases and their specific inhibitors [41]. The secretion of MMP-9 in cell supernatants increased significantly after 24 and $48 \mathrm{~h}$ in comparison with nontreated cells. MMP- 2 secretion was augmented only after $48 \mathrm{~h}$ with incubation of the highest concentrations of nanoparticles $(10$ and $20 \mu \mathrm{g} / \mathrm{mL}$ DNA content). However, zymography studies showed that secreted MMPs were in their proactive form, while in the presence of 10 and $20 \mu \mathrm{g} / \mathrm{mL}$ DNA-containing nanoparticles, the active form of MMP-9, but not MMP-2, was detected in cell lysates. The results obtained were significative only for increased secretion of metalloproteinases, possibly related to nanoparticles phagocytosis.

2.5. Nanoparticles and Stem Cells. Stem cells are lauded for their unique ability to develop into every kind of cell. Even if 
in vivo studies and clinical trials have demonstrated limitations in reconstituting tissue due to the lack of microenvironment-control on proliferation and survival, the successful use of these cells for investigation towards disease therapy is still pursued. Last decade has witnessed a growth in the field of nanoparticles technology for stem cells isolation, maintenance, and regulation: nanoparticles and nano 3D architectures have been developed to control stem cells proliferation, differentiation, and maturation [77]. In terms of skin regeneration, VEGF high-expressing, transiently modified stem cells have been developed for the purpose of promoting angiogenesis [42]. In order to overcome the insufficient expression of angiogenic factors and low cell viability after transplantation, nanotechnology has provided nonviral, biodegradable polymeric nanoparticles to deliver hVEGF gene to human mesenchymal stem cells (hMSCs) and human embryonic stem cell-derived cells (hESdCs). Treated stem cells demonstrated markedly enhanced hVEGF production, cell viability, and engraftment into target tissues. Implantation of scaffolds seeded with VEGF-expressing stem cells (hMSCs and hESdCs) led from 2 to 4 fold higher vessel densities 2 weeks after implantation, compared with control cells or cells transfected with VEGF by using Lipofectamine 2000, a leading commercial reagent. Four weeks after intramuscular injection into mouse ischemic hindlimbs, genetically modified hMSCs substantially enhanced angiogenesis and limb salvage, while reducing muscle degeneration and tissue fibrosis [42]. These results indicate that stem cells engineered with biodegradable polymer nanoparticles may be therapeutic tools for vascularizing tissue constructs and treating ischemic disease.

2.6. Nanofibrous Scaffolds. The basic strategy of engineered tissue regeneration is the construction of a biocompatible scaffold that, in combination with living cells and/or bioactive molecules, replaces, regenerates, or repairs damaged tissues. The scaffold should possess suitable properties, like biocompatibility, controlled porosity and permeability, and, additionally, support for cell attachment and proliferation. This artificial "dermal layer" needs to adhere to and integrate with the wound, which is not always successful for the current artificial dermal analogues available. The high cost of these artificial dermal analogues also makes their application prohibitive both to surgeons and patients. Engineering dermal substitutes with electrospun nanofibres have lately been of prime importance for skin tissue regeneration. Simple electro spinning technology served to produce nanofibrous scaffolds morphologically and structurally similar to the extracellular matrix of native tissues. The engineered network has been shown to support cell adhesion, proliferation, and differentiation mimicking the fibrous architecture of the extracellular matrix [60]. The large surface area and porosity of electrospun nanofibers enables good permeability for oxygen and water and the adsorption of liquids, and concomitantly protects the wound from bacterial penetration and dehydration. This feature shows electrospun nanofibers to be a suitable material for wound dressing, especially for chronic wounds such as diabetic ulcers or burns.
The electro spinning technique can provide both degradable (collagen; chitosan) and nondegradable (PLA, polyvinyl alcohol (PVA) polymers) nanofibers for two-dimensional nanofibrous sheets. Both sorts of biomaterials have been tested in vivo showing an increased rate of wound epithelialisation and dermis organization [34], as well as good antibacterial activity against the Gram-positive and Gram-negative bacteria [35]. Nanofibrous scaffolds of poly(L-lactic acid)-co-poly( $\varepsilon$-caprolactone) (PLACL) and PLACL/gelatin complexes were fabricated by Chandrasekaran et al. [36]. These nanofibres were characterized by fiber morphology, membrane porosity, wettability, and chemical properties by FTIR analysis to culture human foreskin fibroblasts for skin tissue engineering. The results showed that fibroblasts proliferation, morphology, and secretion of collagen were significantly increased in plasma-treated PLACL/gelatin scaffolds compared to PLACL nanofibrous scaffolds. The obtained results proved that the plasma-treated PLACL/gelatin nanofibrous scaffold is a potential biocomposite material for skin tissue regeneration.

Moreover, nanofibrous constructs can be obtained with a 3D profile, even though they scarcely support cells seeding because of their high porosity. Several strategies have been developed to improve cell infiltration, showing promising results [79]. Chong et al. [80] proposed a cost-effective composite consisting of a nanofibrous scaffold directly electrospun onto a polyurethane dressing (Tegaderm, $3 \mathrm{M}$ Medical)_Tegaderm-nanofiber (TG-NF) construct-for dermal wound healing. Cell culture was performed on both sides of the nanofibrous scaffold and tested for fibroblast adhesion and proliferation. Results obtained in this study suggested that both the TG-NF construct and dual-sided fibroblast-populated nanofiber construct achieved significant cell adhesion, growth, and proliferation. This was a successful first step for the nanofiber construct in establishing itself as a suitable three-dimensional scaffold for autogenous fibroblast populations and providing great potential in the treatment of dermal wounds through layered application.

Steps towards an enhanced regenerative effect will be to provide scaffolds a delivery system for drugs, growth factors or cytokines that may further promote cell function and tissue regeneration [81]. Jin et al. already worked towards this direction engineering PLGA microspheres in nanofibrous scaffolds to control the release of PDGF in vivo [37]. PDGF concentration was evaluated in a soft tissue wound repair model in the dorsa of rats. At 3, 7, 14, and 21 days afterimplantation, the scaffold implants were harvested followed by assessments of cell penetration, vasculogenesis, and tissue neogenesis. Gene expression profiles using cDNA microarrays were performed on the PDGF-releasing NFS. The percentage of tissue invasion into microspheres-containing nanofibrous scaffolds at 7 days was higher in the PDGF groups when compared to controls. Blood vessel number in the groups containing either 2.5 or $25 \mu \mathrm{g}$ PDGF was increased above those of other groups at $7 \mathrm{~d}(P<0.01)$. Results from cDNA array showed that PDGF strongly enhanced in vivo gene expression of the CXC chemokine family members such as CXCL1, CXCL2, and CXCL5. Thus, sustained release of rhPDGF-BB, controlled by slow-releasing microspheres 
associated with the nanofibrous scaffold delivery system, enhanced cell migration and angiogenesis in vivo, and may be related to an induced expression of chemokine-related genes. This approach offers a technology to accurately control growth factor release to promote soft tissue engineering in vivo.

\section{Conclusions}

In recent years, nanomedicine has experienced a progressive expansion: its great potential has attracted considerable investments from governments and industry, which are predicted to increase in the near future, leading to further enhancement and development. Similarly, the knowledge of the cellular and molecular processes underlying wound healing has reached a level which let researchers apply for new therapeutic approaches that act directly on cellular and subcellular events during the healing process. Nanotechnology today offers the means to overcome the dimensional barrier of currently used therapies for wounds and ulcers, to reach a dysfunctional molecular target and exert the therapeutic action straight at the origin of the chronic condition. Nanocarriers possess an enormous potential, as nanoparticles-based delivery systems can be highly beneficial to augment the therapeutic power of biological and synthetic molecules. However the promising results brought by new technologies in therapeutics, the real biological effects of nanoparticles have to be carefully assessed before introducing their use in clinical practice. In particular, the release of active peptides may possibly cause interferences with some biological functions and cellular processes. With regard to this, the incorporation of a targeting ligand into nanoparticles has been proposed, in order to give them site-specificity; also, a wide variety of biomaterials has been prompted to meet the specific biological requirements [82]. This could be especially critical for tissue regeneration, where the biomaterial properties could augment the reparative process, or hinder it due to undesirable attributes of the material.

Despite its recognized importance, there have not been systemic studies that probe the targeting efficiency of nanoparticles nor international standards on their toxicology and biocompatibility. Our wish is that further research is to be extended to the applied science of nanotechnology. The revolutionary potential offered by nanoscale therapeutics applied to wound healing involves the need to develop international standards on their biocompatibility. A safe and targeted use is required to prove beneficial to such a world-wide issue, which is chronic wounds and ulcers care.

\section{References}

[1] R. N. Seetharam, "Nanomedicine-emerging area of nanobiotechnology research," Current Science, vol. 91, no. 3, p. 260, 2006.

[2] S. Sandhiya, S. A. Dkhar, and A. Surendiran, "Emerging trends of nanomedicine-an overview," Fundamental and Clinical Pharmacology, vol. 23, no. 3, pp. 263-269, 2009.
[3] S. S. Suri, H. Fenniri, and B. Singh, "Nanotechnology-based drug delivery systems," Journal of Occupational Medicine and Toxicology, vol. 2, no. 1, article no. 16, 2007.

[4] I. L. Medintz, H. Mattousi, and A. R. Clapp, "Potential clinical applications of quantum dots," International Journal of Nanomedicine, vol. 3, no. 2, pp. 151-167, 2008.

[5] B. M. Kuehn, "Chronic wound care guidelines issued," Journal of the American Medical Association, vol. 297, no. 9, pp. 938939, 2007.

[6] W. K. Stadelmann, A. G. Digenis, and G. R. Tobin, "Physiology and healing dynamics of chronic cutaneous wounds," The American Journal of Surgery, vol. 176, no. 2, supplement 1, pp. 26S-38S, 1998.

[7] D. L. Steed, "The role of growth factors in wound healing," Surgical Clinics of North America, vol. 77, no. 3, pp. 575-586, 1997.

[8] D. M. Simpson and R. Ross, "The neutrophilic leukocyte in wound repair a study with antineutrophil serum," Journal of Clinical Investigation, vol. 51, no. 8, pp. 2009-2023, 1972.

[9] M. B. Witte and A. Barbul, "General principles of wound healing," Surgical Clinics of North America, vol. 77, no. 3, pp. 509528, 1997.

[10] D. M. Cooper, "Optimizing wound healing. A practice within nursing's domain," Nursing Clinics of North America, vol. 25, no. 1, pp. 165-180, 1990.

[11] J. W. Madden and E. E. Peacock, "Studies on the biology of collagen during wound healing. I. Rate of collagen synthesis and deposition in cutaneous wounds of the rat," Surgery, vol. 64, no. 1, pp. 288-294, 1968.

[12] N. T. Bennett and G. S. Schultz, "Growth factors and wound healing: part II. Role in normal and chronic wound healing," The American Journal of Surgery, vol. 166, no. 1, pp. 74-81, 1993.

[13] C. Chen, G. S. Schultz, M. Bloch, P. D. Edwards, S. Tebes, and B. A. Mast, "Molecular and mechanistic validation of delayed healing rat wounds as a model for human chronic wounds," Wound Repair and Regeneration, vol. 7, no. 6, pp. 486-494, 1999.

[14] D. R. Yager, S. M. Chen, S. I. Ward, O. O. Olutoye, R. F. Diegelmann, and I. K. Cohen, "Ability of chronic wound fluids to degrade peptide growth factors is associated with increased levels of elastase activity and diminished levels of proteinase inhibitors," Wound Repair and Regeneration, vol. 5, no. 1, pp. 23-32, 1997.

[15] O. Ziv-Polat, M. Topaz, T. Brosh, and S. Margel, "Enhancement of incisional wound healing by thrombin conjugated iron oxide nanoparticles," Biomaterials, vol. 31, no. 4, pp. 741$747,2010$.

[16] W. Hachicha, L. Kodjikian, and H. Fessi, "Preparation of vancomycin microparticles: importance of preparation parameters," International Journal of Pharmaceutics, vol. 324, no. 2, pp. 176-184, 2006.

[17] S. P. Chakraborty, S. K. Sahu, S. K. Mahapatra et al., "Nanoconjugated vancomycin: new opportunities for the development of anti-VRSA agents," Nanotechnology, vol. 21, no. 10, Article ID 105103, 2010.

[18] K. Greenhalgh and E. Turos, "In vivo studies of polyacrylate nanoparticle emulsions for topical and systemic applications," Nanomedicine, vol. 5, no. 1, pp. 46-54, 2009.

[19] E. Turos, J. Y. Shim, Y. Wang et al., "Antibiotic-conjugated polyacrylate nanoparticles: new opportunities for development of anti-MRSA agents," Bioorganic and Medicinal Chemistry Letters, vol. 17, no. 1, pp. 53-56, 2007. 
[20] J. Jain, S. Arora, J. M. Rajwade, P. Omray, S. Khandelwal, and K. M. Paknikar, "Silver nanoparticles in therapeutics: development of an antimicrobial gel formulation for topical use," Molecular Pharmaceutics, vol. 6, no. 5, pp. 1388-1401, 2009.

[21] A. D. Widgerow, "Nanocrystalline silver, gelatinases and the clinical implications," Burns, vol. 36, no. 7, pp. 965-974, 2010.

[22] X. Liu, P. Y. Lee, C. M. Ho et al., "Silver nanoparticles mediate differential responses in keratinocytes and fibroblasts during skin wound healing," ChemMedChem, vol. 5, no. 3, pp. 468$475,2010$.

[23] A. J. Friedman, G. Han, M. S. Navati et al., "Sustained release nitric oxide releasing nanoparticles: characterization of a novel delivery platform based on nitrite containing hydrogel/glass composites," Nitric Oxide, vol. 19, no. 1, pp. 12-20, 2008.

[24] K. Yamasaki, H. D. J. Edington, C. McClosky et al., "Reversal of impaired wound repair in iNOS-deficient mice by topical adenoviral-mediated iNOS gene transfer," Journal of Clinical Investigation, vol. 101, no. 5, pp. 967-971, 1998.

[25] G. Molineux, "Pegylation: engineering improved pharmaceuticals for enhanced therapy," Cancer Treatment Reviews, vol. 28, supplement 1, pp. 13-16, 2002.

[26] O. I. Corrigan and X. Li, "Quantifying drug release from PLGA nanoparticulates," European Journal of Pharmaceutical Sciences, vol. 37, no. 3-4, pp. 477-485, 2009.

[27] T. A. Holland, J. K. V. Tessmar, Y. Tabata, and A. G. Mikos, "Transforming growth factor- $\beta 1$ release from oligo(poly(ethylene glycol) fumarate) hydrogels in conditions that model the cartilage wound healing environment," Journal of Controlled Release, vol. 94, no. 1, pp. 101-114, 2004.

[28] K. Han, K. D. Lee, Z. G. Gao, and J. S. Park, "Preparation and evaluation of poly(L-lactic acid) microspheres containing rhEGF for chronic gastric ulcer healing," Journal of Controlled Release, vol. 75, no. 3, pp. 259-269, 2001.

[29] K. Kawai, S. Suzuki, Y. Tabata, and Y. Nishimura, "Accelerated wound healing through the incorporation of basic fibroblast growth factor-impregnated gelatin microspheres into artificial dermis using a pressure-induced decubitus ulcer model in genetically diabetic mice," British Journal of Plastic Surgery, vol. 58, no. 8, pp. 1115-1123, 2005.

[30] G. Wei, Q. Jin, W. V. Giannobile, and P. X. Ma, "Nano-fibrous scaffold for controlled delivery of recombinant human PDGFBB," Journal of Controlled Release, vol. 112, no. 1, pp. 103-110, 2006.

[31] B. Zavan, V. Vindigni, K. Vezzù et al., "Hyaluronan based porous nano-particles enriched with growth factors for the treatment of ulcers: a placebo-controlled study," Journal of Materials Science: Materials in Medicine, vol. 20, no. 1, pp. 235-247, 2009.

[32] P. L. Bigliardi, D. J. Tobin, C. Gaveriaux-Ruff, and M. BigliardiQi, "Opioids and the skin-where do we stand?" Experimental Dermatology, vol. 18, no. 5, pp. 424-430, 2009.

[33] S. Küchler, N. B. Wolf, S. Heilmann et al., "3D-Wound healing model: influence of morphine and solid lipid nanoparticles," Journal of Biotechnology, vol. 148, no. 1, pp. 24-30, 2010.

[34] M.-S. Khil, D.-I. Cha, H.-Y. Kim, I.-S. Kim, and N. Bhattarai, "Electrospun nanofibrous polyurethane membrane as wound dressing," Journal of Biomedical Materials Research B, vol. 67, no. 2, pp. 675-679, 2003.

[35] C. C. Wang, C. H. Su, and C. C. Chen, "Water absorbing and antibacterial properties of $\mathrm{N}$-isopropyl acrylamide grafted and collagen/chitosan immobilized polypropylene nonwoven fabric and its application on wound healing enhancement," Journal of Biomedical Materials Research A, vol. 84, no. 4, pp. 10061017, 2008.
[36] A. R. Chandrasekaran, J. Venugopal, S. Sundarrajan, and S. Ramakrishna, "Fabrication of a nanofibrous scaffold with improved bioactivity for culture of human dermal fibroblasts for skin regeneration," Biomedical Materials, vol. 6, no. 1, 2011.

[37] Q. Jin, G. Wei, Z. Lin et al., "Nanofibrous scaffolds incorporating PDGF-BB microspheres induce chemokine expression and tissue neogenesis in vivo," PLoS One, vol. 3, no. 3, Article ID e1729, 2008.

[38] L. V. Norling, M. Spite, R. Yang, R. J. Flower, M. Perretti, and C. N. Serhan, "Cutting edge: Humanized nano-proresolving medicines mimic inflammation-resolution and enhance wound healing," Journal of Immunology, vol. 186, no. 10, pp. 5543-5547, 2011.

[39] I.-S. Kim, S.-K. Lee, Y.-M. Park et al., "Physicochemical characterization of poly(l-lactic acid) and poly(d,l-lactide-co-glycolide) nanoparticles with polyethylenimine as gene delivery carrier," International Journal of Pharmaceutics, vol. 298, no. 1, pp. 255-262, 2005.

[40] A. Masotti and G. Ortaggi, "Chitosan micro- and nanospheres: fabrication and applications for drug and DNA delivery," Mini-Reviews in Medicinal Chemistry, vol. 9, no. 4, pp. 463469, 2009.

[41] F. Chellat, A. Grandjean-Laquerriere, R. Le Naour et al., "Metalloproteinase and cytokine production by THP-1 macrophages following exposure to chitosan-DNA nanoparticles," Biomaterials, vol. 26, no. 9, pp. 961-970, 2005.

[42] F. Yang, S.-W. Cho, S. M. Son et al., "Genetic engineering of human stem cells for enhanced angiogenesis using biodegradable polymeric nanoparticles," Proceedings of the National Academy of Sciences of the United States of America, vol. 107, no. 8, pp. 3317-3322, 2010.

[43] S. Werner and R. Grose, "Regulation of wound healing by growth factors and cytokines," Physiological Reviews, vol. 83, no. 3, pp. 835-870, 2003.

[44] G. Lenz and P. Mansson, "Growth factors as pharmaceuticals," Pharmaceutical technology, vol. 15, no. 1, pp. 34-38, 1991.

[45] P. Senet, E. Vicaut, N. Beneton, C. Debure, C. Lok, and O. Chosidow, "Topical treatment of hypertensive leg ulcers with platelet-derived growth factor-BB: a randomized controlled trial," Archives of Dermatology, vol. 147, no. 8, pp. 926-930, 2011.

[46] A. J. Singer and R. A. F. Clark, "Cutaneous wound healing," The New England Journal of Medicine, vol. 341, no. 10, pp. 738746, 1999.

[47] G. Murphy and H. Nagase, "Progress in matrix metalloproteinase research," Molecular Aspects of Medicine, vol. 29, no. 5, pp. 290-308, 2009.

[48] A. MaHam, Z. Tang, H. Wu, J. Wang, and Y. Lin, "Proteinbased nanomedicine platforms for drug delivery," Small, vol. 5, no. 15, pp. 1706-1721, 2009.

[49] D. H. Carney, R. Mann, W. R. Redin et al., "Enhancement of incisional wound healing and neovascularization in normal rats by thrombin and synthetic thrombin receptor-activating peptides," Journal of Clinical Investigation, vol. 89, no. 5, pp. 1469-1477, 1992.

[50] U. Amara, D. Rittirsch, M. Flierl et al., "Interaction between the coagulation and complement system," Advances in Experimental Medicine and Biology, vol. 632, pp. 71-79, 2008.

[51] K. C. Glenn, G. H. Frost, J. S. Bergmann, and D. H. Carney, "Synthetic peptides bind to high-affinity thrombin receptors and modulate thrombin mitogenesis," Peptide Research, vol. 1, no. 2, pp. 65-73, 1988.

[52] M. R. Schäffer, P. A. Efron, F. J. Thornton, K. Klingel, S. S. Gross, and A. Barbul, "Nitric Oxide, and Autocrine Regulator 
of Wound Fibroblast Synthetic Function," The Journal of Immunology, vol. 158, no. 5, pp. 2375-2381, 1997.

[53] R. Weller, R. J. Price, A. D. Ormerod, N. Benjamin, and C. Leifert, "Antimicrobial effect of acidified nitrite on dermatophyte fungi, Candida and bacterial skin pathogens," Journal of Applied Microbiology, vol. 90, no. 4, pp. 648-652, 2001.

[54] L. Englande and A. Friedman, "Nitric oxide nanoparticle technology: a novel antimicrobial agent in the context of current treatment of skin and soft tissue infection," Journal of Clinical and Aesthetic Dermatology, vol. 3, no. 6, pp. 45-50, 2010.

[55] C. Miller, B. McMullin, A. Ghaffari et al., "Gaseous nitric oxide bactericidal activity retained during intermittent highdose short duration exposure," Nitric Oxide, vol. 20, no. 1, pp. 16-23, 2009.

[56] L. R. Martinez, G. Han, M. Chacko et al., "Antimicrobial and healing efficacy of sustained release nitric oxide nanoparticles against staphylococcus aureus skin infection," Journal of Investigative Dermatology, vol. 129, no. 10, pp. 2463-2469, 2009.

[57] N. Barraud, D. Schleheck, J. Klebensberger et al., "Nitric oxide signaling in Pseudomonas aeruginosa biofilms mediates phosphodiesterase activity, decreased cyclic di-GMP levels, and enhanced dispersal," Journal of Bacteriology, vol. 191, no. 23, pp. 7333-7342, 2009.

[58] M. R. Mihu, U. Sandkovsky, G. Han, J. M. Friedman, J. D. Nosanchuk, and L. R. Martinez, "Nitric oxide releasing nanoparticles are therapeutic for Acinetobacter baumannii wound infections," Virulence, vol. 1, no. 2, pp. 62-67, 2010.

[59] A. Y. Peleg, H. Seifert, and D. L. Paterson, "Acinetobacter baumannii: emergence of a successful pathogen," Clinical Microbiology Reviews, vol. 21, no. 3, pp. 538-582, 2008.

[60] P. A. Tran, L. Zhang, and T. J. Webster, "Carbon nanofibers and carbon nanotubes in regenerative medicine," Advanced Drug Delivery Reviews, vol. 61, no. 12, pp. 1097-1114, 2009.

[61] Z. Değim, "Use of microparticulate systems to accelerate skin wound healing," Journal of Drug Targeting, vol. 16, no. 6, pp. 437-448, 2008.

[62] F. Ungaro, G. De Rosa, A. Miro, F. Quaglia, and M. I. La Rotonda, "Cyclodextrins in the production of large porous particles: development of dry powders for the sustained release of insulin to the lungs," European Journal of Pharmaceutical Sciences, vol. 28, no. 5, pp. 423-432, 2006.

[63] K. A. Abbas, A. Mohamed, A. S. Abdulamir, and H. A. Abas, "A review on supercritical fluid extraction as new analytical method," American Journal of Biochemistry and Biotechnology, vol. 4, no. 4, pp. 345-353, 2008.

[64] D. R. Knighton, K. F. Ciresi, and V. D. Fiegel, "Classification and treatment of chronic nonhealing wounds: successful treatment with autologous platelet-derived wound healing factors (PDWHF)," Annals of Surgery, vol. 204, no. 3, pp. 322-330, 1986.

[65] S. Lepidi, G. Abatangelo, V. Vindigni et al., "In vivo regeneration of small-diameter $(2 \mathrm{~mm})$ arteries using a polymer scaffold," The FASEB Journal, vol. 20, no. 1, pp. 103-105, 2006.

[66] N. B. Wolf, S. Küchler, M. R. Radowski et al., "Influences of opioids and nanoparticles on in vitro wound healing models," European Journal of Pharmaceutics and Biopharmaceutics, vol. 73, no. 1, pp. 34-42, 2009.

[67] C. L. Baum and C. J. Arpey, "Normal cutaneous wound healing: clinical correlation with cellular and molecular events," Dermatologic Surgery, vol. 31, no. 6, pp. 674-686, 2005.

[68] J. Hurlow and P. G. Bowler, "Clinical experience with wound biofilm and management: a case series," Ostomy Wound Management, vol. 55, no. 4, pp. 38-49, 2009.
[69] M. Qadan and W. G. Cheadle, "Common Microbial Pathogens in Surgical Practice," Surgical Clinics of North America, vol. 89, no. 2, pp. 295-310, 2009.

[70] S. M. Moghimi, A. C. Hunter, and J. C. Murray, "Long-circulating and target-specific nanoparticles: theory to practice," Pharmacological Reviews, vol. 53, no. 2, pp. 283-318, 2001.

[71] H. J. Klasen, "A historical review of the use of silver in the treatment of burns. II. Renewed interest for silver," Burns, vol. 26, no. 2, pp. 131-138, 2000.

[72] A. Melaiye and W. J. Youngs, "Silver and its application as an antimicrobial agent," Expert Opinion on Therapeutic Patents, vol. 15, no. 2, pp. 125-130, 2005.

[73] J. Fong and F. Wood, "Nanocrystalline silver dressings in wound management: a review," International Journal of Nanomedicine, vol. 1, no. 4, pp. 441-449, 2006.

[74] J. Ai, E. Biazar, M. Jafarpour et al., "Nanotoxicology and nanoparticle safety in biomedical designs," International Journal of Nanomedicine, vol. 6, pp. 1117-1127, 2011.

[75] A. S. Mayo, B. K. Ambati, and U. B. Kompella, "Gene delivery nanoparticles fabricated by supercritical fluid extraction of emulsions," International Journal of Pharmaceutics, vol. 387, no. 1-2, pp. 278-285, 2010.

[76] A. Masotti, F. Bordi, G. Ortaggi, F. Marino, and C. Palocci, "A novel method to obtain chitosan/DNA nanospheres and a study of their release properties," Nanotechnology, vol. 19, no. 5, Article ID 055302, 2008.

[77] X. Yang, J. D. Shah, and H. Wang, "Nanofiber enabled layerby-layer approach toward three-dimensional tissue formation,” Tissue Engineering A, vol. 15, no. 4, pp. 945-956, 2009.

[78] P. A. Tran, L. Zhang, and T. J. Webster, "Carbon nanofibers and carbon nanotubes in regenerativemedicine," Advanced Drug Delivery Reviews, vol. 61, no. 12, pp. 1097-1114, 2009.

[79] C. P. Barnes, S. A. Sell, E. D. Boland, D. G. Simpson, and G. L. Bowlin, "Nanofiber technology: designing the next generation of tissue engineering scaffolds," Advanced Drug Delivery Reviews, vol. 59, no. 14, pp. 1413-1433, 2007.

[80] E. J. Chong, T. T. Phan, I. J. Lim et al., "Evaluation of electrospun PCL/gelatin nanofibrous scaffold for wound healing and layered dermal reconstitution," Acta Biomaterialia, vol. 3, no. 3, pp. 321-330, 2007.

[81] H. S. Yoo, T. G. Kim, and T. G. Park, "Surface-functionalized electrospun nanofibers for tissue engineering and drug delivery," Advanced Drug Delivery Reviews, vol. 61, no. 12, pp. 1033-1042, 2009.

[82] G. Wang and H. Uludag, "Recent developments in nanoparticle-based drug delivery and targeting systems with emphasis on protein-based nanoparticles," Expert Opinion on Drug Delivery, vol. 5, no. 5, pp. 499-515, 2008. 

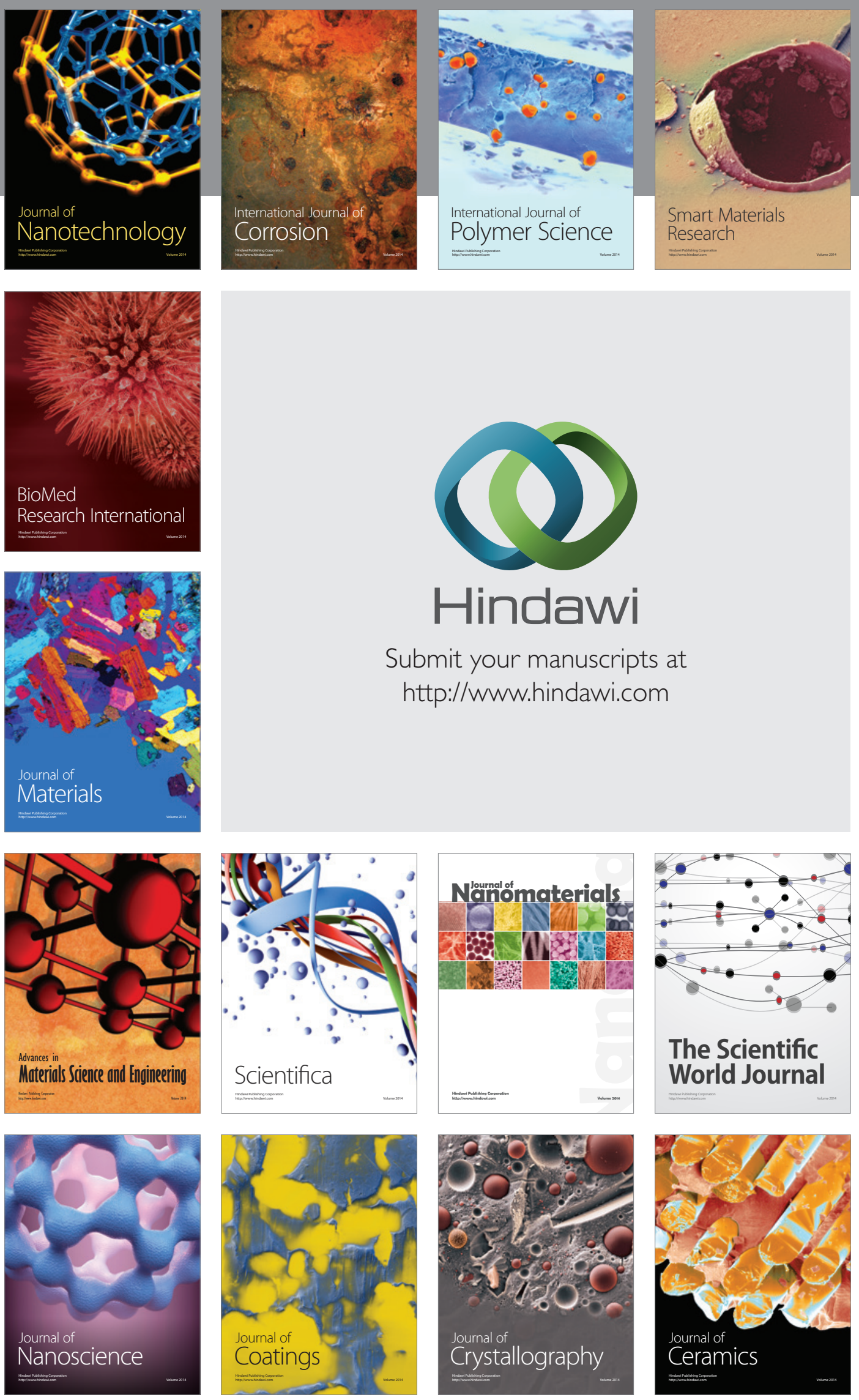

The Scientific World Journal

Submit your manuscripts at

http://www.hindawi.com

\section{World Journal}

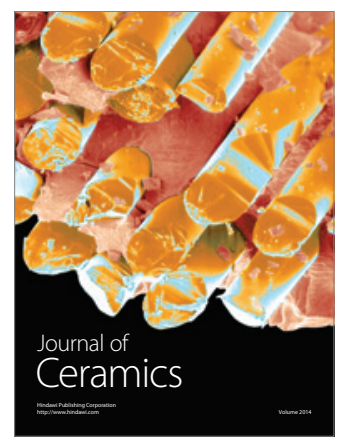

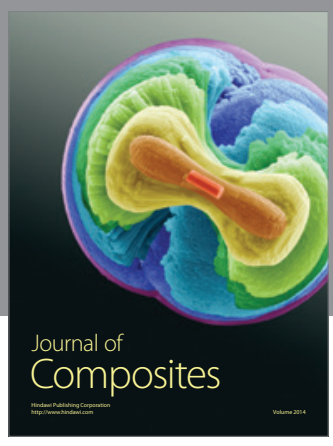
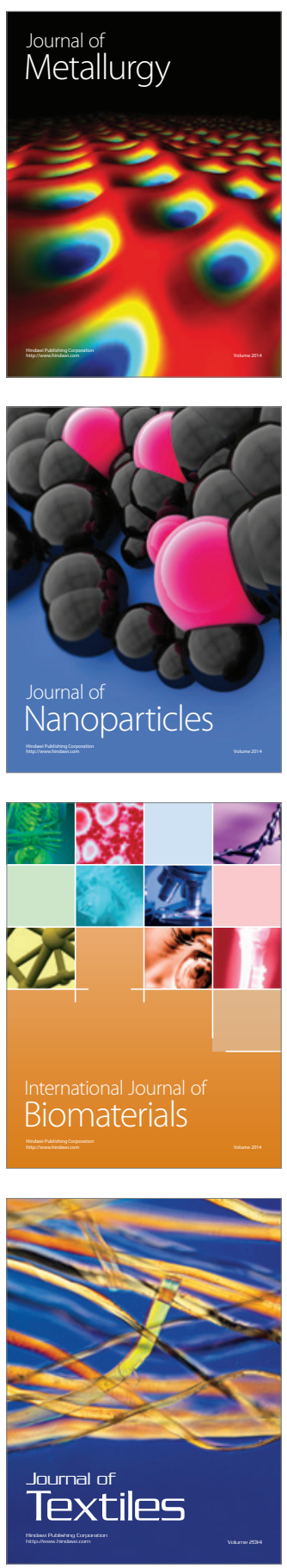